\title{
L'arôme du Camembert : autres composés mineurs mis en évidence
}

\author{
par \\ J. P. DUMONT, Sylviane ROGER et J. ADDA \\ I.N.R.A., Laboratoire de Technologie Laitière - 78350 Jouy-en-Josas
}

\section{INTRODUCTION}

L'arôme du Camembert a donné lieu ces dernières années a une série de publications émanant de deux laboratoires travaillant de façon indépendante avec des méthodes différentes [1, 2, 3].

Les résultats obtenus par ces deux équipes se recoupent en ce qui concerne les composés les plus abondants, mais il est clair que de nombreux autres composés présents à l'état de traces et dont certains peuvent avoir une grande importance organoleptique sont encore susceptibles d'être mis en évidence. C'est ainsi que dans leur dernière publication [3] Moinas et al. ont enrichi de quelques noms (méthylfurylcétone, thiophène-2-aldéhyde, phényl-acétonitrile et propiophénone) la liste des composés déjà répertoriés. Pour notre part il nous a été possible de mettre en évidence un certain nombre de composés non signalés à ce jour en appliquant à des extraits de Camembert la technique de fractionnement récemment utilisée avec succès lors d'une étude sur le Pont l'Evêque [4].

\section{MATERIEL ET METHODES}

Le lot de fromages analysés a été constitué de fromages fabriqués au lait cru. Les fromages non écroûtés ont été râpés à l'état congelé et soumis à une distillation sous vide selon les modalités précédemment décrites [5]. L'extrait aqueux est alors saturé de $\mathrm{NaCl}$, amené à $\mathrm{pH} 9$ et soumis à une série d'extractions par de petites quantités de Fréon 11, puis de dichlorométhane. Après réunion des fractions, chacun des deux extraits est ramené à un volume de quelques $\mathrm{ml}$ en utilisant une colonne adiabatique remplie d'anneaux de Raschig en verre puis concentré jusqu'à un volume de $200 \mu \mathrm{l}$ en utilisant une courte colonne de Dufton à spires de nickel. 
L'extrait Fréon a été fractionné sur colonne d'acide silicique suivant la technique de Palmer [6]. On recueille des fractions de $1 \mathrm{ml}$ dont on évalue pour chacune l'importance olfactive. Les fractions sont ensuite réunies par deux ou trois en fonction de leur caractère olfactif, concentrées et analysées en utilisant le couplage chromatographie en phase gazeuse-spectromètre de masse [2].

L'extrait dichlorométhane est analysé par chromatographie en phase gazeuse-spectrométrie de masse sans fractionnement préalable.

\section{RESULTATS}

Les premières fractions recueillies au cours de la chromatographie de l'extrait Fréon présentaient une odeur qualifiée de vineuse ; l'analyse de ces fractions a permis de montrer qu'elles étaient uniquement constituées d'hydrocarbures qui dans leur totalité avaient déjà été identifiés lors de l'étude précédente.

Les fractions suivantes possédaient une note aillée caractéristique du fromage affiné. L'analyse de ces fractions a conduit à l'identification de plusieurs composés soufrés nouveaux :

- le 2,4-dithiapentane, ou bis(méthylthio)méthane (m/e 61(100) 45(50) 108(34) 35(22) 46(20) 47(17) 63(6) qui avait été improprement identifié dans une précédente publication comme étant le 2,3 dithiapentane ou méthylethyldisulfure ;

- le diéthyldisulfure ou 3,4-dithiahexane (m/e 66(100) 29(94) 122 (54) 94(45) 27(43) 45(18) 59(17) ;

- le 2,4,5-trithiahexane (m/e 61(100) 45(39) 35(23) 46(17) 27(14) 47(12) 63(7) 140(6);

- le II'bis(méthylthio) diméthylsulfure ou 3(méthylthio) 2,4 dithiapentane (m/e 61(100) 45(47) 106(23) 35(18) 46(18) 27(17) 47(15) 154(6).

A ces composés identifiés, il faut ajouter un corps inconnu de formule brute $\mathrm{C}_{8} \mathrm{H}_{14} \mathrm{~S}(\mathrm{~m} / \mathrm{e} 43(100)$ 42(75) 41(70) 47(45) 61(42) 89(33) 27(31) 76(21) 118(18).

Les fractions suivantes correspondent à l'élution des esters et des composés carbonylés. Les qualificatifs utilisés le plus souvent pour les décrire sont : odeurs "florale " ou " poivrée » mais elles ne sont pas apparues comme très caractéristiques du Camembert. Parmi les composés nouveaux identifiés dans ces fractions il faut mentionner l'anisole et deux de ses homologues supérieurs (4-méthylanisole et 2,4-diméthylanisole) ainsi que les esters en $\mathrm{C}_{6}$ et $\mathrm{C}_{\text {s }} \mathrm{du}$ 3-méthyl-1-butanol.

Ces composés toutefois ne sont pas présents dans tous les échantillons analysés. 
L'analyse des fractions suivantes qui comprennent successivement des phénols, des alcools secondaires puis primaires n'a fait que confirmer les résultats précédemment publiés [2].

L'extrait dichlorométhane possédait une odeur fromagère affirmée qui tout en étant caractéristique du Camembert était généralement considérée comme éventée. L'analyse de cet extrait a permis de montrer qu'il était presque uniquement constitué de composés comportant un radical hydroxyle (alcools et phénols), résultat prévisible du fait des polarités respectives du Fréon 11 et du dichlorométhane. En plus des composés déjà répertoriés il a été possible de mettre en évidence :

- le 3-méthylthiopropanol,

- le 3-méthyl-2-cyclohexen-1-ol,

ainsi que deux substances qui n'ont pu être identifiées. On peut toutefois dire que l'une d'entre elles (m/e 79(100) 94(50) 31(10) 45(8) 63(7) 48(7) 81(6) 64(5) est un composé soufré dont le spectre de masse rappelle celui du diméthylsulfone mais qui est probablement doté d'une fonction alcool primaire (m/e 31), l'autre, déjà rencontrée dans le Pont l'Evêque est probablement une amine de masse 115 dont le spectre de masse est $30(100) 43(35) 72(20) 60(13) 58(11) 73(11)$ 100(8) 31(7) 115(5).

\section{DISCUSSION}

Outre la mise en évidence de nouvelles substances volatiles, les résultats présentés ci-dessus permettent d'évaluer la contribution des différentes classes de composés à l'arôme total. Il apparaît en particulier que les hydrocarbures peuvent jouer un rôle dans l'arôme du Camembert comme en témoigne la forte odeur de cave à vin de la fraction qui les contenait. Cette observation rejoint celle de Boyd et al. [8] qui rapportent la présence, dans des fromages Bleus, d'alkyl et d'alkénylbenzènes présentant une odeur d'humus et de bois moisi. Selon ces mêmes auteurs, ces composés importants pour l'arôme des fromages Bleus seraient synthétisés par le Penicillium.

La fraction qui renferme les produits soufrés présente également un grand intérêt tant par son odeur aillée identique à celle de certains Camemberts affinés que par la nature des corps mis en évidence. Il apparaît un certain nombre de composés (bis(méthylthio)méthane, 2, 4,5-trithiahexane et bis(méthylthio)diméthylsulfure) qui pourraient dériver du méthanethiol par condensation ou par cétalisation de ce dernier avec la formaldéhyde. C'est notamment le cas du bis(méthylthio)méthane qui a été mis en évidence dans le Gouda [7]. Cette substance, dont les seuils de perception mesurés dans l'huile et dans l'eau sont extrêmement bas, est considérée par ces auteurs comme un élément fondamental de l'arôme du Gouda. Pour notre part, afin de nous assurer de l'importance réelle de ce composé, nous en déter- 
minons actuellement les seuils de perception olfactifs et gustatifs dans du préfromage obtenu par ultrafiltration, ce substrat étant, à notre sens, plus proche de la réalité fromagère que l'huile ou l'eau. Ces résultats seront publiés ultérieurement.

Le dernier composant identifié de la fraction soufrée est le disulfure d'éthyle. Son origine n'est pas établie, tout au plus peut-on penser qu'il pourrait provenir de la condensation de deux molécules d'éthanethiol, composé qui a déjà été mis en évidence dans le Cheddar [9].

L'extrait dichlorométhane présente une odeur fromagère indéniable quoique très imparfaite sur le plan de l'arôme du Camembert, alors qu'il ne contient qu'un nombre réduit de composés. Ce fait est à rapprocher de l'observation selon laquelle le 3-méthyl-1-butanol, le phényléthanol et le phénol joueraient un rôle important dans l'arôme des fromages à croûte lavée [10]. On voit ainsi se dessiner une base fromagère commune aux fromages à pâte molle, la différenciation entre les différents types pouvant s'effectuer par l'apparition de composés plus spécifiques tels que l'octen-1-ol-3 ou les thioesters par exemple.

Le 3-méthylthiopropanol ou méthionol qui a été mis en évidence à l'état de traces dans l'extrait dichlorométhane provient vraisemblablement de la réduction du méthional, composé qui résulte lui-même de la dégradation et de la désamination de la méthionine. Certains auteurs [11] ont émis l'hypothèse que le méthional dont la présence est généralement transitoire dans les fromages pouvait jouer le rôle de précurseur du méthanethiol et du sulfure de méthyle.

La mise en évidence du méthional pose donc le problème de savoir si ce composé est lui-même un intermédiaire dans la formation du méthanethiol ou s'il s'agit-là d'un produit final comme le sont le phényléthanol et le 3-méthyl-1-butanol qui proviennent eux aussi du métabolisme d'acides aminés.

D'autre part, la présence d'un composé dont le spectre présente une grande similitude avec celui du diméthylsulfone amène à rappeler que ce dernier a déjà été mis en évidence dans le lait [12] et l'huile de beurre [13].

En dehors de ces observations qui ont un rapport direct avec la technique de fractionnement utilisée il est possible de faire deux autres remarques :

L'une concerne l'absence de cinnamate de méthyle, corps qui est pourtant identifié avec régularité par Moinas et al. dans les extraits de Camembert [1]. Dans aucun des échantillons étudiés il n'a été possible de mettre cette substance en évidence et ce, malgré l'obtention d'une fraction ester bien définie.

L'autre remarque a trait à la présence dans une partie des échantillons de l'anisole et de certains de ses dérivés. Ceci est à rapprocher de la mise en évidence du p(1-propenyl)anisole dans le 
Gouda [7]. L'origine et l'importance organoleptique éventuelle de ces composés restent à démontrer.

\section{Rés u m é}

Un certain nombre de composés importants au point de vue de l'arôme mais présents qu'à l'état de traces ont été identifiés dans le Camembert. Il s'agit surtout de composés soufrés et de dérivés de l'anisole. Le spectre de masse de chacun des composés est donné.

\section{S u m m a ry}

Volatile components of Camembert cheese were isolated by low temperature vacuum distillation. Extraction of the aqueous distillate was successively carried out by mean of a non polar and a polar solvent. The non polar extract was fractionated by adsorption chromatography. Combined GC-MS enabled to detect several trace components: bis(methylthio)methane, diethyldisulfide, 2.4.5-trithiahexane, 1,1'bis(methylthio) dimethylsulfide, 3-methylthio-propanol, anisole, 4-methyl anisole and 2.4-dimethyl anisole. The organoleptic importance of some of them is discussed.

Reçu pour publication en mai 1976.

\section{Références}

[1] Moinas (M.), Groux (M.), Horman (I.) (1973). - Le Lait, 53, 529-530, 601.

[2] Dumont (J. P.), Roger (Sylviane), Cerf (Paule), AdDA (J.) (1974). - Le Lait, $54,538,501$.

[3] Moinas (M.), Groux (M.), Horman (I.) (1975). - Le Lait, 55, 547, 414.

[4] Dumont (J. P.), Degas (Christiane), Adda (J.) (1976). - Le Lait, 56, 551.

[5] Dumont (J. P.), Adda (J.) (1972). - Le Lait, 52, 515-516, 311.

[6] Palmer (J. K.) (1973). - J. Agr. Food. Chem., 21, 5, 923.

[7] Sloot (D.), Harkes (P. D.) (1975). - J. Agr. Food Chem., 23, 2, 356.

[8] Boyd (E. N.), Patton (S.), Day (E. A.) (1967). - Cités dans : The Chemistry and Physiology of Flavors. Cheese Flavor. Schultz (H.W.), DAY (E. A.), Libbey (L. M.). AVI Publishing Company.

[9] Liebich (H. M.), Douglas (D. R.), Bager (E.), Zlatkis (A.) (1970). - J. Chromatog. Sci., 8, 355.

[10] Dumont (J. P.), Roger (Sylviane), AdDA (J.) (1974). - Le Lait, 54, 531-532, 31.

[11] DAY (E. A.). - Dans The Chemistry and Physiology of Flavors. Cheese Flavor. Schultz (H. W.), Day (E. A.), LibBey (L. M.). AVI Publishing Company.

[12] Williams (K. I. H.), Burstein (S. H.), Layne (D. S.) (1966). - Proc. Soc Exp. Biol. Med., 122, 865.

[13] Forss (D. A.), Stark (W.), URbach (G.) (1967). - J. Dairy Res., 34, 131. 